\title{
Blood Impedance Characterization from Pulsatile Measurements
}

\author{
Tao Dai \\ School of Information Technology \\ and Engineering (SITE), \\ University of Ottawa, Canada \\ e-mail: tdai@site.uottawa.ca
}

\author{
Andy Adler \\ School of Information Technology \\ and Engineering (SITE), \\ University of Ottawa, Canada \\ e-mail: adler@site.uottawa.ca
}

\begin{abstract}
This paper describes an in vivo measurement technique to calculate dielectric properties of blood excluding the contributions from surrounding tissues, based on analysis of the pulsatile component bioimpedance spectroscopy data. A tetrapolar electrode system applied on the fingers is simulated by a pspice equivalent circuit, and current is injected with frequency ranging from $5 \mathrm{kHz}$ to $100 \mathrm{MHz}$. The finger is modeled as three parts: arterial blood, surrounding tissues and incremental infused arterial blood during systole. The pulsatile curve is fitted to a Cole-Cole model by Levenberg-Marquardt (LM) nonlinear curve fitting. Using the pulsatile impedance data, electrical properties of blood and surrounding tissues can be separated successfully. Parameter accuracies are illustrated according to various noise levels and sampling rates.
\end{abstract}

Keywords - Bioimpedance spectroscopy; pulsatile impedance wave; Cole-Cole model; nonlinear curve fitting.

\section{Introduction}

The bioimpedance measurements in humans have been receiving considerable attentions during the past two decades because of several advantages, such as low cost, easy application, non-invasiveness and on-line monitoring. The original bioimpedance technique was bioelectrical impedance analysis (BIA). Within a decade, this technique evolved into the more advanced technique known as bioelectrical impedance spectroscopy (BIS), also called multiplefrequency bioimpedance analysis (MFBIA). BIS applies multi-frequency stimulations to measure body impedance. This technique has been widely used for many applications, such as:

- Body fluid measurement[1][2]. This method estimates extracellular fluid (ECF), intracellular fluid (ICF) and total body water (TBW);

- Tissue volume change. A typical application of this type is the impedance plethysmography[3].

- Tissue characterization. These techniques are mostly based on Cole-Cole model in terms of Cole-Cole impedance parameters[4]. e.g. in [5], normal and ischemic tissues are differentiated by comparing $r_{0}$ and $f_{c}$. in [6], Zhao et al. calculated plasma resistance, intracellular resistance and cell membrane capacitance of blood using three measuring frequencies.

Characterization of blood bioimpedance properties is of importance for the development of methods estimating some clinical indices such as haematocrit, glucose level, hydration, etc. The ability to make in vivo measurements would be a great advance; however, traditional impedance spectroscopy of blood measurements are in vitro[6][7], or represent an average of all tissues involved in the measurement field, rather than the blood signal alone. In this paper, we propose a novel approach to measure blood impedance in vivo by analysis of data from the cardiac induced pulsatile impedance signal. These data are used to fit a nonlinear model, from which arterial blood parameters are separated from those of the surrounding tissues.

\section{Methods}

A tetrapolar impedance sensor is applied on index finger or between index and middle fingers so that an alternating current enters finger from two injection electrodes and the voltage is measured between two detection electrodes. The physiological structure of this compartment is relatively simple compared with other measuring sites (e.g. chest) and can be approximated with a cylindrical model describe as Fig. 1.

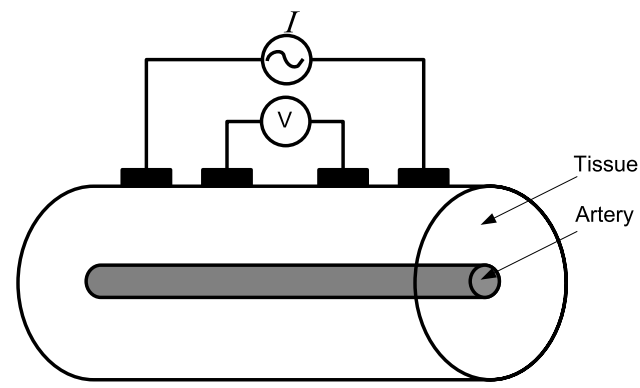

Figure 1. A tetrapolar BIS sensor is applied on a finger segment, modeled as a cylindrical model which contains a uniform blood and tissue compartment.

Artery expansion during heart systole increases its cross section area from $S_{a}$ to $S_{a}+\Delta S_{a}$ and the arterial impedance of this segment decreases correspondingly from $Z_{a}$ to $Z_{a}-$ $\Delta Z_{a}$. The fractional variation is thus $\Delta Z_{a} / Z_{a}=-\Delta S_{a} / S_{a}$. This impedance signal varies with the same frequency as the ECG signal but delayed phase. For simplicity, here we use a sinusoidal wave to simulate the real pulsatile wave (Fig. 2).

In order to build a computational model, we categorize finger tissues into two groups: 1) Tissue, which includes everything except arterial blood (ie. muscle, fat, interstitial fluid, venous and capillary blood), and 2) Arterial blood. There are two situations for arterial blood volume: 1) $D i$ astole: arterial blood volume is minimum, and 2) Systole: maximum arterial expansion. Based on analysis above, the 


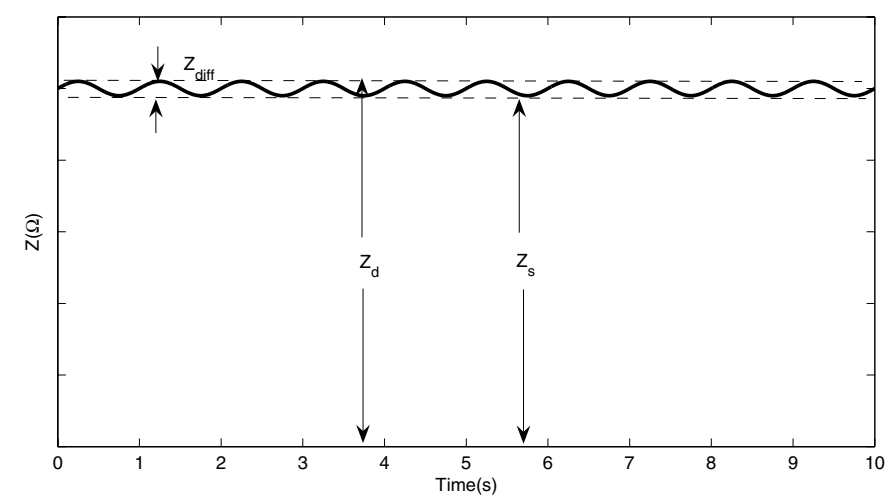

Figure 2. An illustration of the pulsatile impedance wave at a measuring frequency. $Z_{d}$ : impedance (upper dash line) corresponding to the heart diastole; $Z_{s}$ : impedance (lower dash line) corresponding to the maximum arterial expansion during heart systole; $Z_{d i f f}$ : The difference between $Z_{d}$ and $Z_{s}$

pulsatile bioimpedance model of a finger can be described as three electrical components in parallel (Fig. 3).

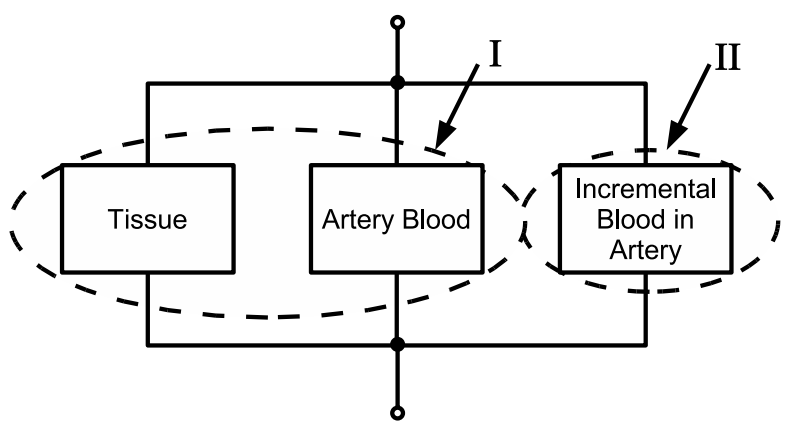

Figure 3. The bioimpedance model of pulsatile wave on a finger, modeled as three parallel impedances. Tissue and arterial blood are grouped as part I (with impedance $Z_{I}$ ), while the incremental blood in the artery is part II (with impedance $Z_{I I}$ ).

The pulsatile impedance measurements $Z_{d}$ and $Z_{s}$ (Fig. 2 ) are modelled to originate from the tissue model (Fig. 3) where tissue and arterial blood are represented by impedance $Z_{I}$ and incremental blood is represented by impedance $Z_{I I}$. During diastole, $Z_{d}=Z_{I}$, while during systole, $Z_{s}=Z_{I} \| Z_{I I}$. During the transition from systole to distribution, $Z_{I I}$ increases as the incremental blood leaves the arteries.

The impedance spectral data are fitted to the Cole-Cole equation [4],

$$
Z(f)=R_{\infty}+\frac{R_{0}-R_{\infty}}{1+j\left(f / f_{c}\right)^{1-a}}
$$

where $R_{\infty}$ is the impedance at infinite frequency, $R_{0}$ is the impedance at zero frequency, and $f_{c}$ is the characteristic frequency of the tissue or model under analysis. $\alpha$ is the constant that characterizes the Cole distribution function. Data are fitted using a nonlinear regression algorithm. The resulting model parameter vector for each tissue group is $m=\left[R_{0}, R_{\infty}, f_{c}, \alpha\right]$.

The Cole-Cole curve of incremental blood (part II, in Fig. 3 ) is calculated as follows:

1. From BIS measurements at diastole, we obtain the ColeCole curve parameters of $Z_{d}(f)=Z_{I}$ (Fig. 4(a), marker $*$ ); 2. From measurements at systole, we obtain the parameters of $Z_{s}(f)=Z_{I} \| Z_{I I}$ by (Fig. 4(a), marker + ).

3. The Cole-Cole curve of $Z_{i b}(f)=Z_{I I}$ (Fig. 4(b), marker $\circ)$ is calculated by

$$
Z_{i b}(f)=\frac{Z_{d}(f) Z_{s}(f)}{Z_{d}(f)-Z_{s}(f)}
$$

The calculated $Z_{i b}(f)$ is blood-related only, based on the model of Fig. 3.

Of the Cole-Cole parameters obtained above, $R_{0}$ and $R_{\infty}$ are geometry-related which are sensitive to body segment dimension changes, electrode movement, and blood pressure changes, etc; $f_{c}$ is relatively stable against body segment geometrical variations; $\alpha$ is insensitive to tissue property variation [5]; Therefore, $f_{c}$ is a good candidate for characterizing blood bioimpedance properties. $\left(R_{0}, R_{\infty}\right.$ and the ratio $R_{0} / R_{\infty}$ are also helpful with careful calibrations)

\section{$3 \quad$ Results}

In order to test this method, we simulate a finger circuit model using a Spice equivalent circuit model with $R, C, L$ components. The model is designed to simulate the physiological structure of a finger and the skin-electrode contact interface, with a frequency sweep of 88 points, logarithmically distributed from $5 \mathrm{kHz}$ to $100 \mathrm{MHz}$. Blood volume in artery is modeled to increase by $10 \%$ during systole. The Cole-Cole curve of incremental blood $\left(Z_{i b}(f), 4(\mathrm{~b})\right)$ is calculated from $Z_{d}(f)$ and $Z_{s}(f)$ (4(a)) through $(2) ; Z_{d}(f)$ (Fig. $4(\mathrm{c})$ ) and $Z_{i b}(f)$ (Fig. $4(\mathrm{~d})$ ) are fitted using the LevenbergMarquardt (LM) nonlinear regression method[8].

Noise analysis is conducted as follows: pusatile impedance is modeled as a sinusoidal wave with amplitude $1 \Omega$ and frequency $1 \mathrm{~Hz}$ on top of a constant pulsatile impedance of $1 \mathrm{k} \Omega$. (Fig. 5(a)); zero-mean, white Gaussian noise with standard deviation $10 \Omega$ is added and the noisy signal is illustrated in Fig. 5(b). $Z_{\text {diff }}$ and $Z_{d}$ are calculated by minimizing a cost function

$$
J=\sum_{i}\left(Z_{d}-\frac{1}{2} Z_{d i f f}+\frac{1}{2} Z_{\text {diff }} f\left(t_{i}\right)-F\left(t_{i}\right)\right)^{2}
$$

where $F(t)$ is the pulsatile impedance wave measured at each frequency, $i=1,2, \ldots, n ; n$ is the number of samples; $f(t)$ is a unit prototype function to mimic pulsatile shape. Here we use a unit amplitude sinusoidal signal as $f(t)$.

The accuracy of the calculated Cole-Cole parameter depend highly on the signal-to-noise ratio and sampling rates. 


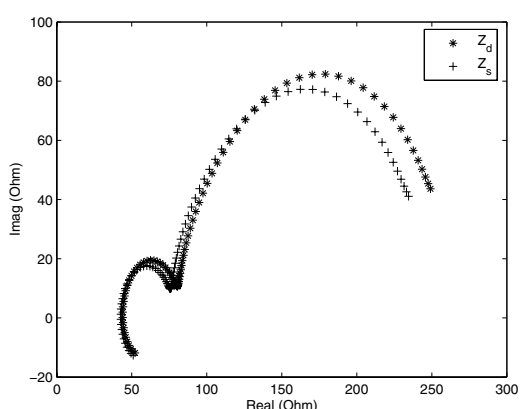

(a)

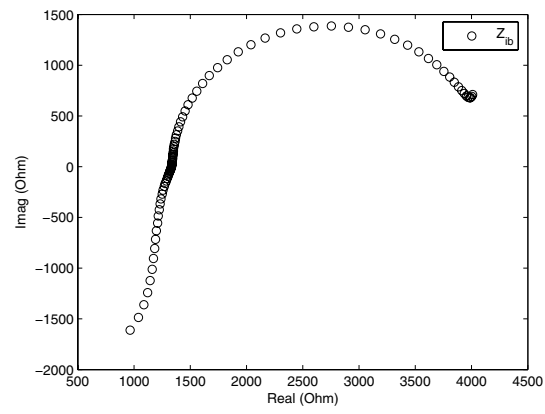

(b)

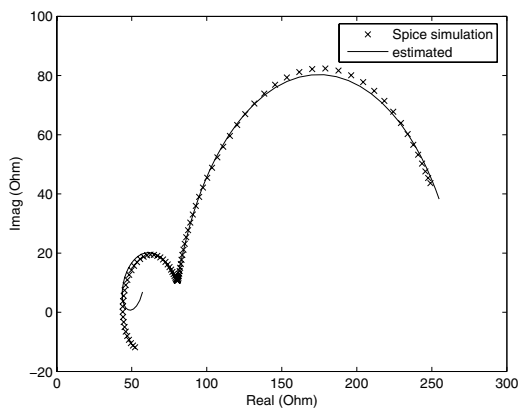

(c)

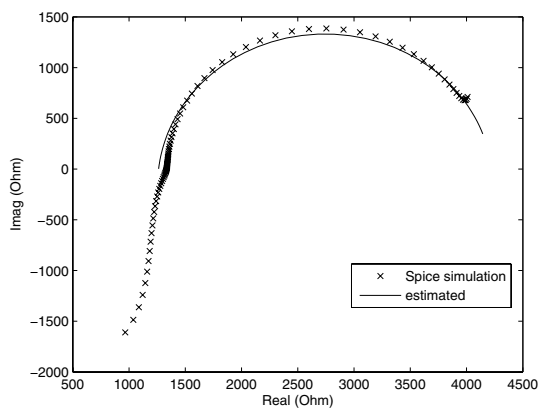

(d)

Figure 4. (a) Cole-Cole curve of $Z_{d}(f)$, and $Z_{s}(f)$, illustrated as ' $*^{\prime}$ and ' ${ }^{\prime}$ ', respectively; (b) pulse curve $Z_{i b}(f)$; where $Z_{d}(f) \| Z_{i b}(f)=Z_{s}(f)$. (c) $Z_{d}(f)$ is fitted in a ECFC model[9]. The Spice simulated data and the fitted data are denoted as ' $\mathrm{x}$ ' and solid lines, respectively. (d) the pulse curve is fitted in a $1^{s t}$ order Cole-Cole model.
Table I shows parameter accuracies as function of SNR, while table II is of sampling rates. Impedance data are collected over 10 seconds per measuring frequency, and statistical results are based on 1000 independent simulations. The errors are evaluated by the square root of Normalized Mean Square Error (NMSE): Err $\_x=\frac{\sigma_{x}}{\mu_{x}}$, in which $\sigma$ and $\mu$ are the standard deviation and mean of the calculated parameters, $\left[Z_{\text {diff }}, Z_{d}, R_{0}, R_{\infty}, f_{c}, \alpha\right]$. Results show parameter errors decrease in proportion to square root of sample number $n, \sigma \propto 1 / \sqrt{n}$. The primary error sources from pulsatile impedance peak-peak amplitude $Z_{d i f f}$; Parameters have various error levels under same experimental conditions $\left(R_{0}\right.$ is the lowest and $\alpha$ is the highest); Decreasing SNR dramatically reduces accuracies.
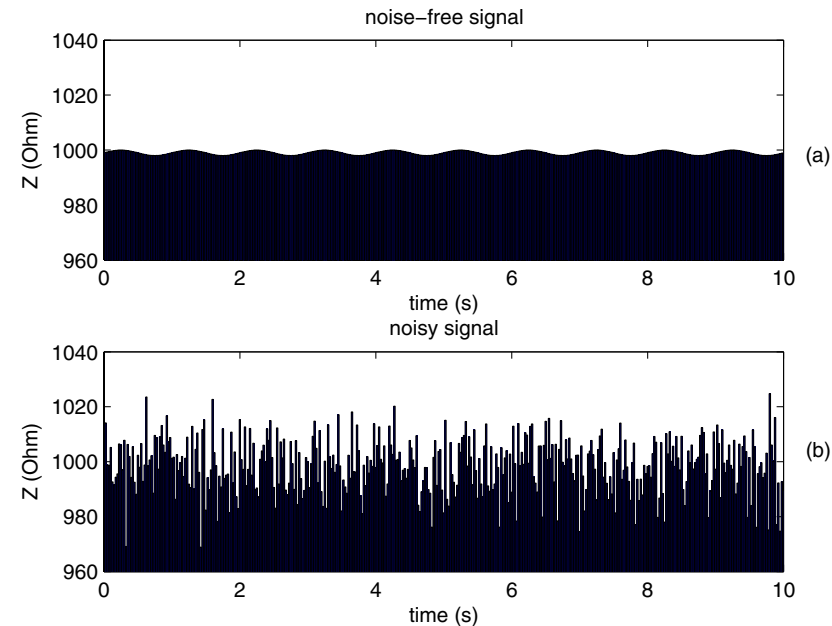

Figure 5. The simulated pulsatile impedance wave. (a) noise free signal; (b) noisy signal. (sampling frequency is $40 \mathrm{~Hz}$ )

TABLE I

Measurement error and corresponding Cole-Cole parameter error, as a function of SNR. (Sampling rate is $1 \mathrm{kHz}$ )

\begin{tabular}{|l|c|c|c|c|}
\hline $\operatorname{SNR}(\mathrm{dB})$ & 0 & -5 & -10 & -20 \\
\hline \hline Err & 1.350 & 2.555 & 4.308 & 13.89 \\
$Z_{\text {diff }}(\%)$ & & & & \\
Err $Z_{d}(\%)$ & 0.0016 & 0.0031 & 0.0054 & 0.011 \\
\hline \hline Err $R_{0}(\%)$ & 0.1911 & 0.3240 & 0.6594 & 1.867 \\
Err $R_{\infty}(\%)$ & 0.5772 & 0.9659 & 1.511 & 5.791 \\
$\operatorname{Err} f_{c}(\%)$ & 0.884 & 1.614 & 2.453 & 8.302 \\
$\operatorname{Err} \alpha(\%)$ & 5.588 & 11.76 & 17.70 & 62.37 \\
\hline
\end{tabular}

\section{Discussion}

This paper introduces a method to measure arterial blood impedance from pulsatile blood impedance measurements. This technique offers the following advantages. In term of physiology, the pulse curve is generated from a homoge- 
TABLE II

Measurement error and corresponding Cole-Cole parameter errors, as a function of sampling rate. ( $S N R=-20 \mathrm{~dB})$

\begin{tabular}{|l|c|c|c|c|c|}
\hline $\begin{array}{l}\text { Sampling } \\
\text { rate }(\mathrm{Hz})\end{array}$ & $10^{1}$ & $10^{2}$ & $10^{3}$ & $10^{4}$ & $10^{5}$ \\
\hline \hline Err & 184.1 & 47.30 & 13.89 & 4.514 & 1.363 \\
$Z_{\text {diff }}(\%)$ & & & & & \\
Err $Z_{d}(\%)$ & 1.001 & 0.030 & 0.011 & 0.003 & 0.0008 \\
\hline \hline Err $R_{0}(\%)$ & 196.7 & 90.60 & 1.867 & 0.605 & 0.184 \\
$\operatorname{Err~} R_{\infty}(\%)$ & 131.3 & 70.06 & 5.791 & 1.865 & 0.514 \\
$\operatorname{Err~} f_{c}(\%)$ & 490.6 & 233.6 & 8.302 & 2.814 & 0.786 \\
$\operatorname{Err} \alpha(\%)$ & 308.9 & 150.3 & 62.37 & 19.27 & 5.661 \\
\hline
\end{tabular}

neous medium - blood. Parameters of this curve, such as $f_{c}$, can be more representative for blood characterization compared with those from curves such as $Z_{d}(f)$ or $Z_{s}(f)$ which are generated from multi-tissues due to heterogeneity in the measuring field. The inductive effect in curves $Z_{d}(f)$ and $Z_{s}(f)$, corresponding to the sub-ripple on the high frequency end (Fig. 4(a)), can be observed in many real measurements even on homogenous tissue[9]. This is alleviated in the pulsatile curve in which, although there still has a extension to negative part of imaginary axis at high frequency, it can be regarded as extrapolation of the Cole-Cole curve without affecting Cole parameters' fitting accuracy. During curve fitting, by using pulse curve, we don't need to adopt additional inductive component (ECFC model[9]) or multiple Cole systems[5][10] due to tissue heterogeneity. A simple $1^{\text {st }}$ order Cole-Cole model is sufficient (Fig. 4(d))which means less computations and higher accuracy of fitted parameters.

The disadvantage of pulse curve is that, in real measurements, the pulsatile amplitude is only a small fraction $(10 \%$ or less) of the noise amplitude which introduces impediments to measure pulsatile amplitude accurately. In order to overcome this, high sampling rates and/or additional signal processing approaches are required which increase computational complexities.

Some previous work is related to the method we propose. Yamakoshi et al.[11] showed that the changes in the admittance produced by pulsatile in the human finger dipped in the electrolyte vanishes when the conductivity of the electrolyte is equal to that of the blood. However, this method is limited by strict experimental conditions. Brown et al.[12] tried to characterize cardiac related impedance wave measured in the chest and found contradictions while comparing resulting cole-cole parameters with those of blood. They demonstrated that the cardiac related impedance wave was not from blood only but a structure like "blood-tissue" parallel pair and this impedance spectrum could be misleading if being directly used for blood characterization.

In conclusion, we propose a new technique to calculate in vivo properties of arterial blood based on measurements of the cardiac induced pulsatile BIS signal in a finger. The measured pulse curve is fitted using nonlinear curve fitting method and a simple first order Cole-Cole model. This method may potentially allow simplified measurement of blood parameters for many biomedical monitoring applications. However, because of noise levels encountered in real measurements, the methodology of obtaining accurate pulse data is concern for the future research and development.

\section{Acknowledgments}

This work is supported by a grant from Ontario Centres of Excellence.

\section{References}

[1] Siconolfi SF, Gretebeck RJ, Wong WW, Pietrzyk RA, Suire SS, "Assessing total body water from bioelectrical response spectroscopy", J Appl Physiol, 82:704710. 1997.

[2] Thomas BJ, Cornish BH, Ward LC "Bioelectrical impedance analysis for measurement of body fluid volumes: A review", J Clin Eng, 17:505-510, 1992.

[3] Nyboer J. Electrical Impedance Plethymography. $\left(2^{\text {nd }}\right.$ edt), Springfield, IL: Thomas, 1970.

[4] Cole KS, Cole RH, "Dispersion and absorption in dielectrics. I. alternating current characteristics", $J$ Chem Phys, 48:341-351, 1941.

[5] Casas O, Bragos R, Riu RJ, Rosell J, Tresanchez M, Warren M, Rodriguez-Sinovas A, Carrena A, Cinca J, "in vivo and in situ ischemic tissue characterization using electrical impedance spectroscopy", Ann NY Acad Sci.20;873:51-8.1999.

[6] Zhao TX, Jacobson B, Ribbe T, "Triple-frequency method for measuring blood impedance," Physiol Meas, 14:145-156, 1993.

[7] Alison JM, Sheppard RJ, "Dielectric properties of human blood at microwave frequencies", Phys Med Biol, 38,971-8, 1993.

[8] Moré JJ, "The Levenberg-Marquardt Algorithm: Implementation and Theory," Numerical Analysis, ed. G. A. Watson, Lecture Notes in Mathematics 630, Springer Verlag, pp. 105-116, 1977.

[9] Lafargue AL, Cabrales LB, Larramendi RM, "Bioelectrical parameters of the whole human body obtained through bioelectrical impedance analysis", Bioelectromagnetics, 23:450-454, 2002.

[10] Grimnes S, Martinsen OG, Bioimpedance \& Bioelectricity Basics. p227. 2000.

[11] Yamakoshi K, Shimazu H, Togawa T, Fukuoka M, Ito H. "Non-invasive measurement of haematocrit by electrical admittance plethysmography technique", IEEE Trans Biomed Eng., 27:156-161. 1980.

[12] Brown BH, Barber DC, Morice AH, Leathard AD, "Cardiac and respiratory related electrical impedance changes in the human thorax", IEEE Trans Biomed Eng, 41:729-34, 1994. 